\title{
Feeding practices and growth among young children during two seasons in rural Ethiopia
}

\author{
Mekitie Wondafrash ${ }^{1,2^{*}}$ D, Lieven Huybregts ${ }^{2,3}$, Carl Lachat ${ }^{2,4}$, Kimberley P. Bouckaert ${ }^{2,4}$ and Patrick Kolsteren ${ }^{2,4}$
}

\begin{abstract}
Background: The use of indices of infant and young child feeding practices to predict growth has generated inconsistent results, possibly through age and seasonal confounding. The aim of this study was to evaluate the association of a dietary diversity score (DDS) and infant and child feeding index (ICFI) with growth among young children in a repeated cross-sectional and a follow-up study in two distinct seasons in rural southwest Ethiopia.

Methods: We used a repeated cross-sectional design comparing child feeding practices to nutritional status in 6-12 month old children during harvest (HS; $n=320)$ and pre-harvest season (PHS; $n=312)$. In addition, 6-12 month old children from the HS were reassessed 6 months later during PHS. In addition to child anthropometry, child feeding practices were collected using 24-h and 7-day dietary recalls.

Results: The mean $( \pm S D)$ length-for-age $z$-score (LAZ) of the 6-12 month old children was $-0.77( \pm 1.4)$ and -1.0 $( \pm 1.3)$ in HS and PHS, respectively, while the mean $( \pm S D)$ of the follow-up children in PHS was $-1.0( \pm 1.3)$. The median DDS (IQR) was $2.0(1.0,3.0), 2.0(2.0,3.0)$ and $3.0(2.0,4.0)$ for the children in HS, PHS and the follow-up children in PHS, respectively. The DDS in HS was positively associated with $L A Z$ at follow-up ( $\beta=0.16 ; 95 \% \mathrm{Cl}$ : 0.01 , $0.30 ; P=0.03$ ) after controlling for confounding factors. ICFI and DDS were not associated with mean $L A Z$, weightfor-height z-score and weight-for-age z-score within season. However, the odds of being stunted when having a DDS $\leq 2$ was 2.3 times (95\% Cl: 1.10, 4.78; $P=0.03$ ) higher compared to a DDS $>2$ child in HS and 1.7 times (95\% Cl: $1.04,2.71 ; P=0.04$ ) higher for the pooled sample of $6-12$ months old children in HS and PHS.

Conclusions: The DDS was found to be an indicator for child stunting during the Ethiopian harvest season. The DDS can be an appropriate tool to evaluate the association of child feeding practices with child growth irrespective of season. Inclusion of other dimensions in the construction of ICFI should be considered in future analysis as we found no association with growth.
\end{abstract}

Keywords: Ethiopia, Dietary diversity, Infant and child feeding index, Growth, Season

\section{Background}

Child undernutrition is persistent in low income countries [1-3]. Ethiopia has one of the highest rates of childhood stunting despite a reduction since 2000 [4]. Among others, suboptimal child feeding practices are important underlying determinants of global poor child growth [5]. Of the children who are breastfed, only a third of children 0-6

\footnotetext{
* Correspondence: mwondafrash@yahoo.com

'Department of Population and Family Health, College of Health Sciences, Jimma University, P.O. Box 343, Jimma, Ethiopia

${ }^{2}$ Department of Food Safety and Food Quality, Faculty of Bioscience Engineering, Ghent University, Coupure Links 653, 9000 Ghent, Belgium Full list of author information is available at the end of the article
}

and 6-23 months old are exclusively breastfed and receive complementary food respectively [4]. Moreover, only $4 \%$ of children 6-23 months old are fed as per the global recommendations.

The construction of summary child feeding indices to assess nutritional outcomes has gained momentum since the early 2000's. The infant and child feeding index (ICFI) was developed based on breast feeding, bottle feeding, feeding frequency and dietary diversity in the previous $24 \mathrm{~h}$ and consumption of food groups in the previous 7 days. It has been suggested that infant and 
child feeding practices tend to cluster in which earlier good practices by a caregiver are more likely to continue later with more awareness on appropriate feeding behaviors [6]. Hence, data on child feeding practices collected over a short period of time were reported to indicate longer term health and nutritional outcomes. On the other hand, a previous review questioned the strength of several existing dietary quality indices in terms of their diagnostic capacity of various health outcomes [7]. Demographic and Health Survey (DHS) data have shown that dietary diversity alone was also associated with height -for-age z-score (HAZ) in 10 out of the 11 countries analyzed [8]. Nevertheless, the associations of infant and child feeding practices with HAZ were not consistent across geographical locations and age categories. The majority of studies showed associations for children aged 12 months and older and not for children in their first year of life. The arbitrary selection of components and cutoffs, as well as determining the contribution of components to the overall index have been highlighted as limitations to predict specific health outcomes or nutritional status [9].

Summary indices that include more information on feeding practices as a whole, in contrast to the diet quality itself, were reported to predict nutritional status [6]. Several evaluations have shown a positive association of such summary indices of child feeding practices with linear growth, i.e. height/length-for-age $\mathrm{z}$ score (HAZ/LAZ) or stunting (HAZ/LAZ <-2) [10-14]. Conversely, the associations between child growth and individual components of the summary feeding index, i.e. breast feeding or bottle feeding alone, were found to be rather incoherent.

Adding to the complexity of associations between dietary quality or feeding practices indicators and child nutritional status are the profound seasonal effects in Sub-Saharan Africa on food availability and accessibility. To our knowledge, no study has examined the impact of seasonality on these associations. Previously, we found an association of DDS with mean micronutrient density adequacy (MMDA) and the association proved to be stable over two distinct seasons in Southwest Ethiopia [15]. Moreover, having a DDS $\leq 2$ was associated with an inadequate intake of micronutrients. The aim of this study was to assess the association of ICFI and DDS with growth among infants and young children. As a first step, we analyzed the associations between ICFI and DDS with child growth using repeated cross-sectional data from two distinct seasons to assess the impact of seasonality on the strength of the association in children 6-12 months of age. Secondly, we assessed the association of ICFI and DDS with child growth longitudinally using follow-up data.

\section{Methods}

\section{Study setting}

The study was conducted in nine kebeles (smaller government unit) of the catchment area of Gilgel Gibe Field Research Center of Jimma University, Southwest Ethiopia. The Research Center was established in 2005 to serve as one of the Demographic Surveillance Systems in Ethiopia and field epidemiology attachment site of Jimma University. According to the census conducted in 2008, the total population within the area was 48,316 of which $49.4 \%$ were males with an average household size of $4.7(\mathrm{SD}=2.2)$. One third of the households were urban [16]. The main source of income for the community is farming and raising livestock. Maize, sorghum and Teff (a species of Eragrostis native to Ethiopia) are commonly grown food crops in the area. Food consumption patterns of the population are expected to be different based on the differences in food availability in the two seasons, i.e. harvest (HS) and pre-harvest (PHS) season.

\section{Sampling and design}

We combined two study designs. Firstly, a repeated cross-sectional survey was conducted using a census of all children aged 6-12 months within the study area during HS, from October to December 2009, and PHS, from June to August 2010 [17]. Children aged 6-12 months old and born in the area, who have a biological mother or female caregiver, presenting no serious illnesses which warrant immediate medical attention or referral (e.g. rapid breathing, nasal flaring, reported high fever, abnormal body movement, persistent diarrhea ( $\geq 2$ weeks) or vomiting, etc.) or malformations affecting anthropometric measurement, and no intention of leaving the area in the coming 6 months were invited to participate. A total of 320 and 312 children were eligible in the HS and PHS, respectively.

Secondly, we conducted a follow-up or cohort study of children who participated in the cross-sectional survey in HS. The follow-up was done 6 months later, i.e. in PHS from June to August 2010 (Fig. 1).

\section{Data collection}

We used pre-tested interviewer-administered questionnaires to collect data on socio-economic status, demographics and infant and young child feeding practices (Additional file 1). Questions were adapted from the Ethiopian Demographic and Health Survey (EDHS) questionnaires [18] and the World Health Organization (WHO) guideline for the assessment of infant and young child feeding [19]. Data collectors with previous experience of data collection in the research center and able to fluently speak the local languages (Amharic and Afan Oromo) were recruited and 


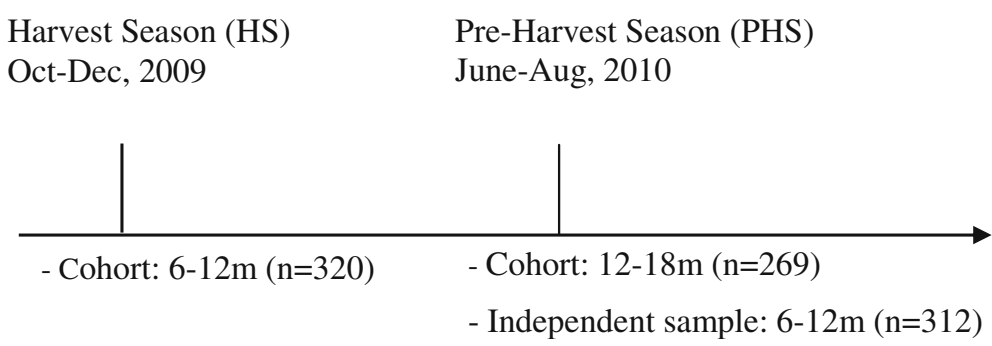

Fig. 1 Study design and age groups of different samples

received a 5-day intensive training from the principal investigators.

\section{Dietary diversity score}

Dietary diversity was measured based on the method suggested by Dewey et al. [20]. The DDS was obtained by summing the number of food groups consumed in the previous $24 \mathrm{~h}$ by the infant. The following seven food groups were included: a) Grains, roots and tubers; b) legumes and nuts; c) dairy products; d) flesh foods; e) eggs; f) vitamin A-rich fruits and vegetables (>130 retinol equivalents/100 g); g) Other fruits and vegetables. Dietary diversity score was used in our analyses as it is or dichotomized. Previous analyses showed that a DDS $\leq 2$ is associated with a low micronutrient intake [15, 20, 21] which warrants the investigation of its association with growth of infants and young children.

\section{Construction of the infant and child feeding index}

The ICFI was calculated using core indicators as described previously [21, 22]. Dietary diversity was assessed using the method described above and age-specific scores were assigned (Table 1). As there was no information on energy density of complementary foods or breast milk intake, the scoring given to feeding frequency is based on the previous recommendation [23] which

Table 1 Scoring system used to create the infant and child feeding index for children aged 6-12 months

\begin{tabular}{lll}
\hline Components & \multicolumn{2}{l}{ Age-specific scores } \\
\cline { 2 - 3 } & $6-8$ months & $9-12$ months \\
\hline Breast feeding & Yes $=2$ & Yes $=2$ \\
No $=0$ & No $=0$ \\
Bottle feeding & Yes $=0$ & Yes $=0$ \\
No $=1$ & No $=1$ \\
Dietary diversity (24 h) & $0-1=0$ & $0-2=0$ \\
& $2=1$ & $3=1$ \\
Food group frequency (previous 7 days) & $0-2=0$ & $0-3=0$ \\
& $3-4=1$ & $4-5=1$ \\
Feeding frequency & $5+=2$ & $6+=2$ \\
& $0-1=0$ & $0-2=0$ \\
& $2=1$ & $3=1$ \\
& $3+=2$ & $4+=2$ \\
\hline
\end{tabular}

assumes low energy density and average breast milk intake. A score of +1 was attributed for those who met the lower end of the recommendation and +2 for those who exceeded the recommendation. Food group frequency was assessed using a questionnaire developed specifically for this purpose in which we asked the number of days a specific food group was consumed. Frequency was then scored 0 if not consumed during the previous week, +1 if consumed on 1-3 days and +2 if consumed for $\geq 4$ days. These scores were summed up yielding a possible range of $0-14$. Subsequently, new scores were assigned depending on age category as shown in Table 1. Each component of ICFI was defined and scored according to the current age-specific infant and young child feeding recommendations [24]. The ICFI was calculated by adding up all the age-specific scores. The index was cut into tertiles in which a score of $0-5$ was considered low, 6-7 as medium and 8-9 as high, as described previously by Moursi et al. [13]. The index was also treated as a continuous variable in our analyses.

\section{Anthropometry}

Anthropometric measurements were standardized through repetitive exercises. Recumbent length was measured on an infant measuring board and recorded to the nearest $0.1 \mathrm{~cm}$ (SECA 210, Hamburg, Germany). Child's weight was measured naked or with very light clothes using mother-child digital scales and recorded to the nearest $0.1 \mathrm{~kg}$ (SECA Uniscale, Hamburg, Germany). Age was recorded from birth certificates or immunization cards as much as possible. If reliable documents for age estimation were absent, local events calendars were used to help the mother or caregiver estimate the approximate age of the child. Most of the anthropometric data collectors in HS were also available in PHS.

\section{Household socio-economic status}

We constructed an asset-based proxy to estimate the socio-economic status (SES) of the included households. Items included were ownership of cattle, house construction materials, land (homestead, land under cultivation, fallow land) and ownership of other household assets. For 
household assets, categories for each item were 'owned' or 'not owned' by the household. The first principal component (56\% of the variance) from a principal components analysis was divided in tertiles and used as a proxy for SES [25].

\section{Morbidity}

Morbidity was assessed qualitatively by asking the mother about the presence of diarrhea (defined as $\geq 3$ episodes of loose stool per day), fever and cough (yes/no) in the previous 2 weeks of the survey.

\section{Data analysis}

The anthropometric measures were converted into $\mathrm{z}$ scores of weight-for-age (WAZ), length-for-age (LAZ) and weight-for-length (WLZ) based on the WHO 2006 Child Growth Standards [26]. Underweight, wasting and stunting were defined as WAZ, WLZ and LAZ $<-2$ $\mathrm{Z}$-scores from the median of the standard, respectively [27]. Descriptive analyses were conducted for SES and demographic data, morbidity, maternal schooling, household characteristics, nutritional status, dietary diversity and feeding practices of children aged 6-12 months for the repeated cross-sectional study. Demographic data, morbidity, nutritional status and dietary diversity were described separately for the follow-up children aged 1218 months.

Means $( \pm$ SD) were reported for continuous data which were normally distributed, otherwise the median (IQR) was used. Differences in means were statistically tested using a student t-test for normally distributed outcomes or Wilcoxon-Mann-Whitney test for skewed outcomes, while a $\chi^{2}$-square test was used to compare categorical variables. The DDS was used as a standalone outcome and as a component of ICFI in the analyses. We used multiple linear regression models to analyze the association between DDS and ICFI with LAZ, WLZ and WAZ adjusted for SES and demographic data, morbidity status, maternal schooling of the 6-12 month old children in the repeated cross-sectional studies and follow-up children. Multiple logistic regressions were used to model DDS and ICFI with binary categories of LAZ, WLZ and WAZ (cut-off value: $-2 \mathrm{SD}$ ). Bivariate analyses were performed between all predictor variables and anthropometric outcomes and then every variable with a modest association $(P<0.25)$ was a candidate to be included in the multivariate analyses. A full model assessing the relation between DDS, ICFI and components of ICFI was compiled after which non-significant variables were removed by a backward procedure using a likelihood ratio test $(P<0.05)$.

The internal consistency of the ICFI was checked by using both the Fisher exact test of association of ICFI (categorized) with its components being bottle feeding, food group frequency and feeding frequency, and by the Cronbach $\alpha$ coefficient.

As there was no effect of season on the association of DDS and ICFI and child growth or nutritional status, the data of the two cross-sectional studies were pooled and the same analysis was performed to increase power adjusting for season and the abovementioned confounding variables. As the interaction of DDS or ICFI with season was not significant $(P>0.1)$, the interaction term was not included in the models. Moreover, a DDS cutoff of $\leq 2$ was used to dichotomize DDS based on a prior sensitivity and specificity analysis [15] and its association with child anthropometry was tested.

ANCOVA was used to assess the longitudinal relationship of anthropometric indices with DDS and ICFI in the follow-up children [28]. For this purpose anthropometric indices at follow-up (PHS) were used as outcome variables and anthropometric indices at baseline (HS) as predictor variables. The DDS and ICFI at HS were also entered separately in the models as predictor variables. Data was analyzed with Stata 13.0 (StataCorp, College Station, TX, USA). Statistical significance was set at 5\% and all tests were two-sided.

\section{Results}

In the repeated cross-sectional surveys in HS and PHS, data was collected from 320 and 312 children aged 6-12 months respectively. In total, 24 children in HS and 21 children in PHS who had incomplete complementary food intake data and/or information pertaining to breast feeding ( $\mathrm{HS}=21$; PHS $=15)$ or uncertain age $(\mathrm{HS}=3$; PHS =6) were omitted from analysis. The mean LAZ, WAZ and WLZ of excluded observations did not differ statistically from the rest of the observations in both seasons.

Of the 296 children with complete data at HS, 27 (9\%) children could not be traced after repeated visits at follow-up. Three observations from the follow-up children were excluded from analysis as they did not have complete dietary diversity data.

\section{Nutritional status}

In the repeated cross-sectional surveys in HS and PHS, 6-12 month old children had a stunting prevalence of 18.6 and $21.1 \%$ respectively, and a wasting prevalence of 11.8 and $15.5 \%$ respectively. The mean LAZ was $-0.77 \pm$ 1.4 and $-1.0 \pm 1.3$ for the children in HS and PHS respectively. At follow-up, $21 \%$ of $12-18$ month old children were stunted and $20 \%$ were wasted, whereas the mean LAZ was $-1.0 \pm 1.3$ (Table 2).

\section{Child feeding practices}

All children aged 6-12 months were partially breastfed at the time of the surveys. The median (IQR) recalled 
Table 2 Characteristics of 6-12 month old children in the repeated cross-sectional and follow-up study in HS and PHS

\begin{tabular}{|c|c|c|c|c|}
\hline Variables & $\begin{array}{l}\text { HS } \\
(n=296)\end{array}$ & $\begin{array}{l}\text { PHS } \\
(n=291)\end{array}$ & $P$-value ${ }^{*}$ & $\begin{array}{l}\text { Follow- up at PHS } \\
(n=266)\end{array}$ \\
\hline \multicolumn{5}{|l|}{ Gender of child } \\
\hline Female & $154(52.0)$ & $125(43.0)$ & 0.03 & $137(51.5)$ \\
\hline Age of the child (6-12) & & & & - \\
\hline $6-8$ months & $152(51.4)$ & $71(24.4)$ & $<0.001$ & \\
\hline 9-12 months & $144(48.6)$ & $220(75.6)$ & & \\
\hline \multicolumn{5}{|l|}{ Age of the child (12-18) } \\
\hline 12-15 months & & & & $183(68.8)$ \\
\hline 16-18 months & & & & $83(31.2)$ \\
\hline Age of the mother & & & & - \\
\hline$<20$ years & $60(20.6)$ & $32(11.0)$ & ${ }^{<} 0.01$ & \\
\hline 20-34 years & $202(69.4)$ & $233(80.1)$ & & \\
\hline$\geq 35$ years & $29(10.0)$ & $26(8.9)$ & & \\
\hline Socioeconomic status, n (\%) & & & & - \\
\hline Low & $79(27.3)$ & $115(39.9)$ & 0.01 & \\
\hline Medium & $106(36.7)$ & 89 (30.9) & & \\
\hline High & $104(36.0)$ & $84(29.2)$ & & \\
\hline Under-five children in the $\mathrm{HH}, n(\%)$ & & & & - \\
\hline $0-1$ & $79(26.7)$ & $66(22.7)$ & 0.26 & \\
\hline$\geq 2$ & $217(73.3)$ & $225(77.3)$ & & \\
\hline \multicolumn{5}{|l|}{ Morbidity, n (\%) } \\
\hline Cough $^{a}$ & $44(14.9)$ & $57(19.6)$ & 0.13 & $36(13.5)$ \\
\hline Fever $^{\mathrm{a}}$ & $57(19.3)$ & $67(23.0)$ & 0.27 & $53(19.9)$ \\
\hline Diarrhea $^{a}$ & $49(16.7)$ & $70(24.1)$ & 0.03 & $67(25.2)$ \\
\hline Source of drinking water for $\mathrm{HH}, n(\%)$ & & & & - \\
\hline Unsafe water source & $252(85.1)$ & $229(78.7)$ & 0.04 & \\
\hline \multicolumn{5}{|l|}{ Human waste disposal by the $\mathrm{HH}, n(\%)$} \\
\hline Improper disposal & $117(39.7)$ & $97(33.3)$ & 0.11 & \\
\hline Maternal schooling, $n(\%)$ & & & & - \\
\hline No formal schooling & $243(82.1)$ & $246(84.5)$ & 0.43 & \\
\hline$L A Z$, mean $\pm S D$ & $-0.77 \pm 1.4$ & $-1.0 \pm 1.3$ & 0.02 & $-1.0 \pm 1.3$ \\
\hline \multicolumn{5}{|l|}{ Stunting, $n(\%)$} \\
\hline$L A Z<-2$ & 55 (18.6) & $61(21.1)$ & 0.44 & $55(20.7)$ \\
\hline$L A Z<-3$ & $18(6.1)$ & $16(5.5)$ & 0.78 & $16(6.0)$ \\
\hline $\mathrm{WLZ}$, mean $\pm \mathrm{SD}$ & $-0.63 \pm 1.3$ & $-0.75 \pm 1.3$ & 0.28 & $-0.81 \pm 1.2$ \\
\hline \multicolumn{5}{|l|}{ Wasting, $n(\%)$} \\
\hline $\mathrm{WLZ}<-2$ & $35(11.8)$ & $45(15.5)$ & 0.19 & $51(19.2)$ \\
\hline $\mathrm{WLZ}<-3$ & $12(4.1)$ & $12(4.1)$ & 0.96 & $11(4.1)$ \\
\hline$W A Z$, mean $\pm S D$ & $-0.96 \pm 1.2$ & $-1.1 \pm 1.4$ & 0.08 & $-1.1 \pm 1.1$ \\
\hline \multicolumn{5}{|l|}{ Underweight, n (\%) } \\
\hline$W A Z<-2$ & $52(17.6)$ & $75(25.8)$ & 0.02 & 49 (18.4) \\
\hline$W A Z<-3$ & $18(6.1)$ & $20(6.9)$ & & $13(4.9)$ \\
\hline
\end{tabular}


Table 2 Characteristics of 6-12 month old children in the repeated cross-sectional and follow-up study in HS and PHS (Continued)

\begin{tabular}{|c|c|c|c|c|}
\hline \multicolumn{5}{|c|}{ Current breast feeding } \\
\hline Yes & $296(100)$ & $291(100)$ & - & $253(95.1)$ \\
\hline DDS, median (IQR) & $2.0(1.0,3.0)$ & $2.0(2.0,3.0)$ & 0.17 & $3.0(2.0,4.0$ \\
\hline ICFI, median (IQR) & $5.0(5.0,7.0)$ & $6.0(5.0,7.0)$ & 0.09 & - \\
\hline
\end{tabular}

HS harvest season, $P H S$ pre-harvest season, $H H$ household, $L A Z$ length-for-age z-score, $W L Z$ weight-for-length z-score, WAZ weight-for-age z-score, $D D S$ Dietary diversity score, ICFI Infant and Child Feeding Index

${ }^{*} P$-values are calculated for the difference of the two independent samples at HS and PHS

a Diarrhea, cough, fever in the previous 2 weeks of the studies

duration of exclusive breastfeeding was $4.0(3.0,4.0)$ and 4.0 (3.0, 6.0) months in HS and PHS respectively; while 19 and $11 \%$ of these children were bottle-fed during HS and PHS respectively. Eighty-eight and sixty seven percent $(P<0.05)$ of the children were introduced to complementary foods before 6 months of age in HS and PHS respectively. There was no significant difference in DDS $(P=0.17)$ and ICFI $(P=0.09)$ between the two seasons $(P=0.17)$ for children aged 6-12 months (Table 2).

The follow-up data showed that breastfeeding was continued well into the second year of life. Only $4.8 \%$ of the follow-up children were not breastfed. The median DDS (IQR) was $3.0(2.0,4.0)$ (Table 2).

\section{Internal consistency of the components of ICFI for the cross-sectional study}

The ICFI was positively associated with dietary diversity, food group frequency and feeding frequency and bottle feeding $(P<0.001)$ in the repeated cross-sectional study but not with breastfeeding as all were breastfed. However, the overall internal consistency estimated by the Cronbach- $\alpha$ coefficient was low $(\alpha=0.45$ in HS and $\alpha=$ 0.47 in PHS), but slightly better in the age group of $6-8$ months in PHS $(\alpha=0.58)$.

\section{Association between feeding practices and child nutrition Repeated cross-sectional study}

The ICFI and DDS were not associated with LAZ and WHZ among 6-12 month old children in both HS and PHS. After each component of the ICFI was assessed separately for its association with LAZ and WHZ, only bottle-feeding was associated with LAZ in PHS $(\beta=$ $-0.74,95 \%$ CI: $-1.20,-0.28 ; P=0.002$ ) in favor of those who were bottle fed (Table 3 ). Children with a DDS $\leq 2$ account for 68 and $63 \%$ of all children in HS and PHS respectively. The odds of being stunted for children with a DDS $\leq 2$ was 2.3 (95\% CI: $1.1,4.8 ; P=0.03)$ times higher compared to their peers with DDS $>2$ during the HS.

In view of the absence of an important seasonal impact on the association between DDS, IFCI and child nutrition, we pooled the data of 6-12 month old children from the two seasons to increase power when testing the association of DDS and ICFI with nutritional status of children.
We observed that the odds of being stunted were 1.70 (95\% CI: 1.04, 2.71; $P=0.04$ ) times higher among children with a DDS $\leq 2$ compared to those with a DDS $>2$. Moreover, being 9-12 months old [OR = 2.0; 95\% CI: 1.24, 3.18; $P<0.01]$, having diarrhea $[\mathrm{OR}=1.70$; $95 \% \mathrm{CI}: 1.05,2.78$; $P=0.04]$ and the presence of $\geq 2$ under five children in the same household [OR $=2.3 ; 95 \%$ CI: $1.24,4.46 ; P=0.01]$ were significantly associated with stunting (Table 4).

\section{Follow-up study}

The DDS of the 6-12 months old children during HS was positively associated with LAZ at follow-up period during PHS ( $\beta=0.16$; 95\% CI: 0.01, 0.30; $P=0.03$ ) (Table 5). The occurrence of diarrhea in the same season was negatively associated with LAZ $(\beta=-0.35$; 95\% CI: $-0.67,-0.03 ; P=$ 0.03). However, the DDS was not associated with LAZ and WHZ within seasons for the follow-up children.

\section{Discussion}

The dietary diversity score during the HS was found to be an independent predictor of the subsequent 6 months of linear growth. Stunting was found to be associated with low DDS $(\leq 2)$ only during HS. Moreover, we did not find such an association for ICFI. Within seasons, we did not find cross-sectional associations between DDS and ICFI and mean LAZ, WHZ and WAZ.

We previously found that children who consumed $\leq 2$ food groups were likely to have inadequate micronutrient intake [15]. In the present analysis, the odds of being stunted was significantly higher for those who consumed $\leq 2$ food groups in the previous $24 \mathrm{~h}$. This was observed both among children in the HS and in the pooled data. Previous analyses also showed that DDS of $\leq 2$ was an indicator of a low nutrient dense diet [20, 29]. An evaluation of the association of DDS with child anthropometry was not performed taking a DDS of $\leq 2$ as a cutoff in those previous analyses. In one study in Mali, children in urban households with lowest DDS had higher risk of stunting and underweight [30]. Ruel and Arimond used tertiles of dietary diversity and the association with HAZ was observed in many of the countries for which the analyses was performed using DHS data [8]. Furthermore, it was observed that significant proportions of children from the African countries included 
Table 3 Associations of ICFI and its components with LAZ and WLZ of children in the repeated cross-sectional study ${ }^{\text {b }}$

\begin{tabular}{|c|c|c|c|c|c|c|c|c|c|c|}
\hline \multirow[t]{2}{*}{ Variables } & \multicolumn{5}{|c|}{ Harvest season $(n=296)$} & \multicolumn{5}{|c|}{ Preharvest season $(n=291)$} \\
\hline & $\bar{n}$ & Mean LAZ (SD) & $P$-value ${ }^{*}$ & Mean WLZ (SD) & $P$-value ${ }^{*}$ & $n$ & Mean LAZ (SD) & $P$-value ${ }^{*}$ & Mean WLZ (SD) & $P$-value ${ }^{*}$ \\
\hline \multicolumn{11}{|l|}{ Tertile of ICFI } \\
\hline Low (reference) & 152 & $-0.86(1.41)$ & & $-0.66(1.26)$ & & 132 & $-1.04(1.23)$ & & $-0.78(1.42)$ & \\
\hline Medium & 97 & $-0.66(1.43)$ & 0.63 & $-0.59(1.30)$ & 0.39 & 119 & $-1.20(1.48)$ & 0.41 & $-0.70(1.36)$ & 0.21 \\
\hline High & 47 & $-0.74(1.35)$ & 0.78 & $-0.63(1.26)$ & 0.50 & 40 & $-0.60(1.25)$ & 0.40 & $-0.80(0.99)$ & 0.96 \\
\hline \multicolumn{11}{|c|}{ Dietary diversity $(24 h)^{a}$} \\
\hline Low (reference) & 140 & $-0.77(1.47)$ & & $-0.65(1.25)$ & & 157 & $-1.13(1.28)$ & & $-0.75(1.34)$ & \\
\hline Medium & 99 & $-0.81(1.38)$ & 0.17 & $-0.62(1.37)$ & 0.50 & 75 & $-1.18(1.51)$ & 0.72 & $-0.87(1.36)$ & 0.74 \\
\hline High & 57 & $-0.66(1.31)$ & 0.60 & $-0.63(1.13)$ & 0.42 & 59 & $-0.64(1.18)$ & 0.27 & $-0.61(1.33)$ & 0.43 \\
\hline \multicolumn{11}{|c|}{ Food group frequency $(7 d)^{a}$} \\
\hline Low (reference) & 62 & $-0.95(1.51)$ & & $-0.67(1.11)$ & & 34 & $-1.22(1.31)$ & & $-0.79(1.55)$ & \\
\hline Medium & 75 & $-0.74(1.52)$ & 0.46 & $-0.84(1.39)$ & 0.66 & 52 & $-1.09(1.41)$ & 0.78 & $-0.74(1.30)$ & 0.93 \\
\hline High & 159 & $-0.72(1.31)$ & 0.28 & $-0.52(1.26)$ & 0.52 & 205 & $-1.01(1.31)$ & 0.24 & $-0.74(1.32)$ & 0.96 \\
\hline \multicolumn{11}{|l|}{ Bottle feeding $^{a}$} \\
\hline Yes (reference) & 56 & $-0.75(1.43)$ & & $-0.68(1.29)$ & & 32 & $-0.21(1.22)$ & & $-0.25(1.15)$ & \\
\hline No & 240 & $-0.78(1.41)$ & 0.54 & $-0.62(1.26)$ & 0.39 & 259 & $-1.15(1.31)$ & 0.002 & $-0.80(1.35)$ & 0.15 \\
\hline \multicolumn{11}{|l|}{ Feeding frequency ${ }^{a}$} \\
\hline Low (reference) & 147 & $-0.88(1.40)$ & & $-0.67(1.30)$ & & 158 & $-1.09(1.21)$ & & $-0.85(1.41)$ & \\
\hline Medium & 63 & $-0.55(1.24)$ & 0.18 & $-0.36(1.19)$ & 0.05 & 54 & $-1.12(1.47)$ & 0.44 & $-0.48(1.51)$ & 0.07 \\
\hline High & 86 & $-0.76(1.53)$ & 0.92 & $-0.78(1.25)$ & 0.87 & 79 & $-0.91(1.50)$ & 0.79 & $-0.74(1.03)$ & 0.61 \\
\hline
\end{tabular}

ICFI Infant and child feeding index, LAZ length-for-age z-score, WLZ weight-for-length z-score

${ }^{*} P$-values are from multiple linear regression models controlling for confounding variables using backward stepwise selection. The association of individual components and LAZ and WLZ were modeled separately

${ }^{a}$ The low, medium and high categories are based on the scoring given for the each component of infant and child feeding index as $0,+1$ and +2 respectively as described in Table 1

${ }^{\mathrm{b}}$ All analyses are adjusted for child and maternal characteristics and household socioeconomic status in the multivariate regression model

in the analysis were consuming $\leq 2$ food groups. A Kenyan study found an association between dietary diversity, calculated from 3 days average consumption of unique food groups, and five anthropometric indices. However, contrary to the present study, the samples of these previous studies were heterogeneous in terms of age groups and breast feeding status, which might explain the inconsistent results of associations between DDS and growth [31]. Younger children are more dependent on breast milk and their complementary food lack diversity compared to older children [32].

We did not observe a significant association between ICFI and LAZ after adjusting for confounders. This was also reported previously by studies conducted in rural West Africa [33] and China [11]. This finding, however, is in contrast to many other studies which showed significant associations of ICFI with LAZ [10, 12-14, 34]. Prior to the adoption of the WHO IYCF measurement indicators, the grouping of food items was done differently apart from differences in the number of food groups used to construct the DDS [35, 36], a component of ICFI. The numbers of components used to construct ICFI were also not uniform over different studies. For instance, we used five components whereas Sawadogo et al. [14], Ntab et al. [33] and Zhang et al. [11] used six, seven and eight components to construct the feeding index, respectively. Ntab et al. used an age group of 1242 months for the analysis of the association between the ICFI and LAZ [33]. Moreover, dietary diversity is an important component in determining the relationship with child anthropometry when summary feeding measures are used $[10,14,21]$. The majority of the children (67\%) in the present study consumed few food groups which in turn can affect ICFI's discriminatory power and hence its association with LAZ [37]. All children in this study were breastfed and were given a high score for the breast feeding component in the construction of the ICFI. The latter scenario might have diluted the association of the ICFI with LAZ in comparison to a setting with a mixture of breastfed and non-breastfed children. Moreover, other studies have shown that breast feeding continues for an extended period of time in sick or malnourished children [38-41] who in turn were given a high score for the summary index.

There was no significant association between DDS and ICFI during the HS and PHS in 6-12 months old children 
Table 4 Predictors of stunting in the pooled sample of all 6-12 months old children participating in the repeated cross-sectional study $^{a}$

\begin{tabular}{|c|c|c|c|c|}
\hline Variables & $n^{c}(\%)$ & Odds Ratio & $95 \% \mathrm{Cl}$ & $P$-value \\
\hline \multicolumn{5}{|l|}{$\overline{\mathrm{DDS}}$} \\
\hline $\mathrm{DDS}>2$ & $204(34.8)$ & Reference & & \\
\hline $\mathrm{DDS} \leq 2$ & $383(65.2)$ & 1.7 & $1.04,2.71$ & 0.04 \\
\hline \multicolumn{5}{|l|}{ Season } \\
\hline $\mathrm{HS}$ & $296(50.4)$ & Reference & & \\
\hline PHS & $291(49.6)$ & 1.0 & $0.64,1.59$ & 0.91 \\
\hline \multicolumn{5}{|l|}{ Human waste disposal } \\
\hline Improper disposal & $214(36.5)$ & Reference & & \\
\hline Proper disposal & $373(63.5)$ & 0.7 & $0.42,1.01$ & 0.05 \\
\hline \multicolumn{5}{|l|}{ Maternal schooling } \\
\hline No formal schooling & $489(83.3)$ & Reference & & \\
\hline Attended formal school & $98(16.7)$ & 0.5 & $0.25,1.07$ & 0.07 \\
\hline \multicolumn{5}{|l|}{ Age of the child } \\
\hline $6-8$ months & $223(38.0)$ & Reference & & \\
\hline 9-12 months & $364(62.0)$ & 2.0 & $1.24,3.18$ & $<0.01$ \\
\hline \multicolumn{5}{|l|}{ Age of the mother } \\
\hline$<20$ years & $92(15.8)$ & Reference & & \\
\hline 20-34 years & $435(74.7)$ & 0.6 & $0.36,0.98$ & 0.03 \\
\hline$\geq 35$ years & $55(9.5)$ & 0.7 & $0.27,1.58$ & 0.35 \\
\hline \multicolumn{5}{|l|}{ Diarrhea $^{b}$} \\
\hline No diarrhea & $466(79.7)$ & Reference & & \\
\hline Diarrhea present & $119(20.3)$ & 1.7 & $1.05,2.78$ & 0.04 \\
\hline
\end{tabular}

Under-five children in the $\mathrm{HH}$

\begin{tabular}{lllll}
$0-1$ & $145(24.7)$ & Reference & & \\
$\geq 2$ & $442(75.3)$ & 2.3 & $1.24,4.46$ & 0.01 \\
\hline
\end{tabular}

DDS dietary diversity score, $H S$ harvest season, $P H S$ pre-harvest season, $H H$ household

${ }^{a}$ Results from multiple binary logistic regression models using a backward stepwise selection of predictors

${ }^{\mathrm{b}}$ Diarrhea in the previous 2 weeks of the studies

'Total numbers included in the analysis. Due to missing observations, the numbers for predicator variables "Age of the mother" and "Diarrhea" do not add up to 587

participating in the repeated cross-sectional study. However, we found that DDS in HS was an independent predictor of linear growth during the 6 months of follow-up. This has also been observed by other researchers using a longitudinal ICFI (a summary indicator from repeated ICFIs over time) rather than an ICFI measured at the time of child anthropometry assessment. Such a composite ICFI was found to be associated with LAZ in children who were followed up for 7 months [21]. Another study in urban China showed that a longitudinal ICFI, but not dietary diversity, was associated with LAZ measured 1 year later [34]. This might indicate that of the positive evolution of feeding practices which led to positive changes in child anthropometry in the long term. DDS, rather than
Table 5 Multiple regression assessing the association between DDS and Length-for-Age Z-score adjusting for relevant confounding factors among the follow-up children in $\mathrm{PHS}^{\mathrm{a}}$

\begin{tabular}{lllll}
\hline Variables & $n^{c}(\%)$ & $\beta$ & $95 \% \mathrm{Cl}$ & $P$-value \\
\hline DDS during HS & 266 & 0.16 & $0.01,0.30$ & 0.03 \\
HAZ during HS & 266 & 0.33 & $0.23,0.43$ & $<0.001$ \\
Age of the mother & & & & \\
$\quad<20$ years & $54(21.1)$ & Reference & & \\
20-34 years & $181(68.3)$ & 0.43 & $0.01,0.80$ & 0.02 \\
>35 years & $27(10.6)$ & 0.66 & $0.04,0.13$ & 0.01 \\
Diarrhea & & & & \\
$\quad$ No diarrhea & $199(74.8)$ & Reference & & \\
Diarrhea Present & $67(25.2)$ & -0.35 & $-0.67,-0.03$ & 0.03 \\
Maternal schooling & & & & \\
No formal schooling & $217(81.6)$ & Reference & & \\
Attended formal school & $49(18.4)$ & 0.33 & $-0.05,0.71$ & 0.05 \\
Socio-economic status & & & & \\
Low & $71(26.9)$ & Reference & & \\
Medium & $94(35.6)$ & 0.41 & $0.06,0.77$ & 0.04 \\
High & $99(37.5)$ & 0.25 & $-0.10,0.60$ & 0.24
\end{tabular}

DDS Dietary diversity score, HAZ height-for-age z-score, HS harvest season ${ }^{a}$ Analysis of covariance was performed to determine predictors of growth at follow-up

${ }^{\mathrm{b}}$ Diarrhea in the previous 2 weeks of the follow up study

'Total numbers included in the analysis. Due to missing observations, the numbers for predicator variables "Age of the mother" and "Socioeconomic status" do not add up to 266

ICFI, was a predictor of growth for the follow-up children in the present study, but it is known that dietary diversity is an important factor in defining the relationship of ICFI with child anthropometry. Nevertheless, optimal growth is more of a cumulative effect of feeding practices involving several nutrients on top of other factors such as household food security, environmental hygiene and healthcare $[3,6$, $10,31,36,42]$.

Overall, child feeding practices in the present study are suboptimal compared to international recommendations with 88 and $67 \%$ of study children being introduced to complementary foods before the recommended age of 6 months during the HS and PHS respectively. Bottle feeding was relatively common and associated with LAZ in favor of bottle fed. On the contrary, premature introduction of complementary foods negatively affected linear growth in a longitudinal study among Vietnamese children [43]. According to an Ethiopian national survey, non-breastfed children were more likely to be fed with solid and semi-solid foods compared with breastfed children [4] but its association with growth was not elucidated. Breastfeeding for an extended period time was proposed to be associated with low HAZ in rural Senegal after mothers delayed weaning for stunted children [40, 41]. A stronger association was also found 
between dietary diversity and HAZ in non-breastfed children compared to breastfed peers $[8,38]$. However, we were not able to demonstrate such associations as all of our children were breastfed.

This study has a number of strengths, but also several limitations that need to be addressed. First, the study was conducted in two distinct seasons in which intake of nutrients and LAZ scores significantly differed, which adds to the external validity of the overall analysis. Secondly, we followed children from the HS to another season to assess clustering of child feeding practices and its effect on growth in the long term. However, the internal validity of the components of the ICFI was found to be lower than what has been arbitrarily accepted. It is also impossible to rule out the day-to-day variability, random or reporting error in the assessment of child feeding practices which can result in misclassification and a reduced precision to detect an association [6]. Over-reporting of desirable feeding behaviors is a common occurrence in the measurement of feeding practices using recall techniques [6]. As it has been demonstrated previously [21, 34], it could have been more appropriate to construct the ICFI from feeding practice data collected from the same subjects at different time points to evaluate the occurrence of clustering of feeding behaviors and their impact on growth or nutritional status in the long term.

\section{Conclusions}

In conclusion, DDS and ICFI are not unequivocally related to child growth. Whereas DDS during the HS was able to predict longer term growth and only very poor dietary diversity was found to be associated with child stunting during HS and in the pooled data. Our data suggests the use of a minimal DDS $(\leq 2)$ rather than a mean DDS as an indicator for suboptimal child growth. We did not find any association between ICFI and child growth, which might suggest that there is a room to add other dimensions of appropriate practices of complementary feeding, such as responsiveness and hygienic preparation and storage of complementary foods.

\section{Additional file}

Additional file 1: Survey questionnaire. Questionnaire administered to the study participants to collect data on feeding patterns and practices and child growth. (DOC $130 \mathrm{~kb}$ )

\footnotetext{
Abbreviations

DDS: Dietary diversity score; EDHS: Ethiopia Demographic and Health Survey; HAZ: Height-for-age z-score; HH: Household; HS: Harvest Season; ICFI: Infant and child feeding index; IQR: Interquartile range; LAZ: Length-for-age z-score; MMDA: Mean micronutrient density adequacy; OR: Odds ratio; PHS: Preharvest season; SD: Standard deviation; SES: Socioeconomic status; WAZ: Weight-for-age z-score; WHO: World Health Organization; WHZ: Weight-for-height z-score; WLZ: Weight-for-length z-score
}

\section{Acknowledgments}

We thank field supervisors, data collectors and mothers and the zonal and local administration. We are also grateful for the contribution of the Gilgel Gibe Field Research Center (GGFRC) of Jimma University for providing background information to conduct the census in the study area.

\section{Funding}

This study is part of the collaborative multidisciplinary research being done between Flemish Universities (Belgium) and Jimma University (Ethiopia). The study was financed by VLIR-UOS through the Institutional University Cooperation Program of Jimma University (IUC-JU). LH received funding from the CGIAR Research Program on Agriculture for Nutrition and Health $(\mathrm{A} 4 \mathrm{NH})$, led by the International Food Policy Research Institute. The funding organizations had no role in the design of the study, data collection and analysis, interpretation and write up of the manuscript.

\section{Availability of data and materials}

The datasets used and/or analyzed during the current study are available from the corresponding author on reasonable request.

\section{Authors' contributions}

MW, PK \& KB: designed the study, supervised if the data was collected as per the protocol, and contributed to the actual implementation of the study. MW acquired the data from the field. LH, CL supported in the analysis and interpretation of the data. MW, the corresponding author, drafted the manuscript. All authors have read and approved the final manuscript.

\section{Authors' information \\ MW: currently working at the Department of Population and Family Health, College of Health Sciences, Jimma University (Ethiopia) and affiliated to the Department of Food Safety and Food Quality, Faculty of Bioscience Engineering, Ghent University (Belgium); CL, KB \& PK: academic staff members at the Department of Food Safety and Food Quality, Faculty of Bioscience Engineering, Ghent University and Unit of Nutrition and Child Health, Institute of Tropical Medicine (Belgium); LH: A research fellow at Poverty, Nutrition and Health Division, International Food Policy Research Institute (USA) and affiliated to Department of Food Safety and Food Quality, Faculty of Bioscience Engineering, Ghent University (Belgium).}

\section{Competing interests}

The authors declare that they have no competing interests.

\section{Consent for publication}

Not applicable.

Ethics approval and consent to participate

The study was approved by the Ethics Review Board of Jimma University, Ethiopia (RPGC/104/2002) and the Ethics Committee of Ghent University Hospital, Belgium (B67020109188). Informed consent was secured from the mothers/caretakers before conducting the interviews and assessments.

\section{Publisher's Note}

Springer Nature remains neutral with regard to jurisdictional claims in published maps and institutional affiliations.

\section{Author details}

'Department of Population and Family Health, College of Health Sciences, Jimma University, P.O. Box 343, Jimma, Ethiopia. ${ }^{2}$ Department of Food Safety and Food Quality, Faculty of Bioscience Engineering, Ghent University, Coupure Links 653, 9000 Ghent, Belgium. ${ }^{3}$ Poverty, Health and Nutrition Division, International Food Policy Research Institute, 2033 K Street NW, 20006 Washington DC, USA. ${ }^{4}$ Unit of Nutrition and Child Health, Institute of Tropical Medicine, Nationalestraat 155, 2000 Antwerp, Belgium. 


\section{Received: 17 August 2016 Accepted: 21 April 2017}

\section{Published online: 24 April 2017}

\section{References}

1. Bhutta ZA, Das JK, Rizvi A, Gaffey MF, Walker N, Horton S, Webb P, Lartey A, Black RE. Evidence-based interventions for improvement of maternal and child nutrition: what can be done and at what cost? Lancet. 2013:382(9890):452-77.

2. Ahmed T, Mahfuz M, Ireen S, Ahmed AMS, Rahman S, Islam MM, Alam N, Hossain MI, Rahman SMM, Ali MM, et al. Nutrition of children and women in Bangladesh: trends and directions for the future. J Health Popul Nutr. 2012;30(1):1

3. Lutter CK, Daelmans BMEG, de Onis M, Kothari MT, Ruel MT, Arimond M, Deitchler M, Dewey KG, Bloessner M, Borghi E. Undernutrition, poor feeding practices, and low coverage of key nutrition interventions. Pediatrics. 2011;128(6):e1418.

4. Central Statistical Agency (CSA) [Ethiopia], ORC Macro. Ethiopia demographic and health survey 2011, Addis Ababa, Ethiopia and Calverton, Maryland, USA: Central Statistical Agency and ORC Macro. 2011.

5. UNICEF. Infant and young child feeding, programming guide; nutrition section, programmes, United Nations Children's Fund, New York. 2012.

6. Ruel MT, Arimond M: Measuring childcare practices: approaches, indicators, and implications for programs, vol. 6: Intl Food Policy Res Inst; Washington, D.C. 2003

7. Kourlaba G, Panagiotakos DB. Dietary quality indices and human health: a review. Maturitas. 2009;62(1):1-8.

8. Arimond $M$, Ruel M. Dietary diversity is associated with child nutritional status: evidence from 11 demographic and health surveys. J Nutr. 2004;134:2579-85.

9. Kant AK. Dietary patterns and health outcomes. J Am Diet Assoc. 2004; 104(4):615-35.

10. Ruel M, Menon P. Child feeding practices are associated with child nutritional status in Latin America: innovative uses of the demographic and health surveys. J Nutr. 2002;132:1180-7.

11. Zhang J, Shi L, Wang J, Wang Y. An infant and child feeding index is associated with child nutritional status in rural China. Early Hum Dev. 2009;85(4):247-52.

12. Bork K, Cames CC, Barigou S, Cournil A, Diallo A. A summary index of feeding practices is positively associated with height-for-age, but only marginally with linear growth, in Rural Senegalese infants and toddlers. J Nutr. 2012;142(6):1116-22.

13. Moursi MM, Treche S, Martin-Prevel Y, Maire B, Delpeuch F. Association of a summary index of child feeding with diet quality and growth of 6-23 months children in urban Madagascar. Eur J Clin Nutr. 2009:63(6):718-24.

14. Sawadogo P, Martin-Prevel $Y$, Savy M, Kameli $Y$, Traissac P, Traore A, Delpeuch F. An infant and child feeding index is associated with the nutritional status of 6- to 23-month-old children in rural Burkina Faso. J Nutr. 2006;136:656-63.

15. Wondafrash M, Huybregts L, Lachat C, Bouckaert KP, Kolsteren P: Dietary diversity predicts dietary quality regardless of season in 6-12-month-old infants in south-west Ethiopia. Public Health Nutrition 2016, 19(14):24852494

16. Institute of Health Science Research (Jimma University). Population and household heads' demographics, policy brief number 1, Jimma, Ethiopia. 2011.

17. Famine and Early Warning Systems Network (FEWS NET): Seasonal calendar in a typical year 2016. http://www.fews.net/east-africa/ethiopia/foodsecurity-outlook/october-2015. Accessed 28 Nov 2016.

18. Central Statistical Agency (CSA) [Ethiopia], ORC Macro. Ethiopia demographic and health survey 2005. Addis Ababa, Ethiopia and Calverton, Maryland, USA: CSA and ORC. 2006

19. WHO. WHO Anthro for personal computers, version 2, 2007: software for assessing growth and development of the world's children. Geneva. 2007.

20. Dewey KG, Cohen RJ, Arimond M, Ruel MT. Developing and validating simple indicators of complementary food intake and nutrient density for breastfed children in developing countries. Washington, DC: the Food and Nutrition Technical Assistance (FANTA) Project, Academy for Educational Development (AED); 2006.

21. Moursi MM, Martin-Prevel Y, Eymard-Duvernay S, Capon G, Treche S, Maire $B$, Delpeuch F. Assessment of child feeding practices using a summary index: stability over time and association with child growth in urban Madagascar. Am J Clin Nutr. 2008;87(5):1472-9.
22. Arimond M, Ruel MT. Progress in developing an infant and child feeding index: an example using the Ethiopia demographic and health survey 2000 2002. In.: Discussion Paper.

23. $\mathrm{PAHO} / \mathrm{WHO}$. Guiding principles for complementary feeding of the breastfed child. Division of health promotion and protection. Food and nutrition program: Washington, DC. 2003.

24. World Health Organization. Indicators for assessing infant and young child feeding practices: part II measurement.: Geneva WHO. 2010.

25. Vyas S, Kumaranayake L. Constructing socio-economic status indices: how to use principal components analysis. Health Policy Plan. 2006;21(6):459-68.

26. WHO. WHO Anthro for personal computers, version 3.2.2, 2011: Software and macros for assessing growth and development of the world's children. Geneva (http://www.who.int/childgrowth/software/en/). 2010.

27. $\mathrm{WHO}$. WHO child growth standards: length/height-for-age, weight-for-age, weight-for-length, weight-for-height and body mass index-for-age. Methods and development. In: WHO Child Growth Standards: Length/Height-for-age, weight-for-age, weight-for-length, weight-for-height and body mass indexfor-age. 2006

28. Twisk JW. Applied longitudinal data analysis for epidemiology: a practical guide. Cambridge, UK; Cambridge University Press; 2003

29. Moursi M, Arimond M, Dewey K, Treche S, Ruel M, Delpeuch F. Dietary diversity is a good predictor of the micronutrient density of the diet of 6- to 23-month-old children in Madagascar. J Nutr. 2008;138:2448-53.

30. Hatløy A, Hallund J, Diarra MM, Oshaug A. Food variety, socioeconomic status and nutritional status in urban and rural areas in Koutiala (Mali). Public Health Nutr. 2000;3(01):57-65.

31. Onyango AW. Dietary diversity, child nutrition and health in contemporary African communities. Comp Biochem Physiol A Mol Integr Physiol. 2003; 136(1):61-9

32. Onyango AW, Receveur O, Esrey SA. The contribution of breast milk to toddler diets in western Kenya. Bull World Health Organ. 2002;80:292-9.

33. Ntab B, Simondon K, Milet J, Cisse B, Sokhna C, Boulanger D, Simondon F. A young child feeding index is not associated with either height-for-age or height velocity in rural Senegalese children. J Nutr. 2005;135:457-64.

34. Ma J-Q, Zhou L-L, Hu Y-Q, Liu J-R, Liu S-S, Zhang J, Sheng X-Y. A summary index of infant and child feeding practices is associated with child growth in urban Shanghai. BMC Public Health. 2012;12(1):568.

35. Marriott BP, White A, Hadden L, Davies JC, Wallingford JC. World Health Organization $(\mathrm{WHO})$ infant and young child feeding indicators: associations with growth measures in 14 low-income countries. Matern Child Nutr. 2012;8(3):354-70.

36. Jones AD, Ickes SB, Smith LE, Mbuya MN, Chasekwa B, Heidkamp RA, Menon P, Zongrone AA, Stoltzfus RJ. World Health Organization infant and young child feeding indicators and their associations with child anthropometry: a synthesis of recent findings. Matern Child Nutr. 2014;10(1):1-17.

37. Disha A, Rawat R, Subandoro A, Menon P. Infant and Young Child Feeding (IYCF) practices in Ethiopia and Zambia and their association with child nutrition: analysis of demographic and health survey data. Afr J Food Agric Nutr Dev. 2012;12(2):5895-914.

38. Marquis GS, Habicht JP, Lanata CF, Black RE, Rasmussen KM. Association of breastfeeding and stunting in Peruvian toddlers: an example of reverse causality. Int J Epidemiol. 1997;26(2):349-56.

39. Simondon KB, Simondon F. Mothers prolong breastfeeding of undernourished children in rural Senegal. Int J Epidemiol. 1998;27(3):490-4.

40. Simondon KB, Costes R, Delaunay V, Diallo A, Simondon F. Children's height, health and appetite influence mothers' weaning decisions in rural Senegal. Int J Epidemiol. 2001;30(3):476-81.

41. Simondon KB, Simondon F, Costes R, Delaunay V, Diallo A. Breast-feeding is associated with improved growth in length, but not weight, in rural Senegalese toddlers. Am J Clin Nutr. 2001;73(5):959-67.

42. Black R, Allen L, Bhutta Z, Caulfield L, de Onis M, Ezzati M. Maternal and child undernutrition: global and regional exposures and health consequences. Lancet. 2008;371:243-60.

43. Hop LT, Gross R, Giay T, Sastroamidjojo S, Schultink W, Lang NT. Premature complementary feeding is associated with poorer growth of vietnamese children. J Nutr. 2000:130(11):2683-90. 